\title{
Behavior of Spent LWR Fuel Decay Heat
} -For Better Insight into Fukushima-Daiichi Accident-

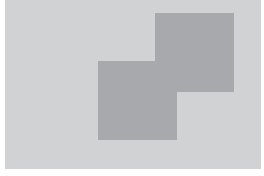

\author{
Tokyo City University, Tadashi Yoshida
}

The struggle with decay heat is considered to be a focal problem that can be attributed to the cold shutdown of the Fukushima Daiichi Nuclear Power Plant. The decay heat is broadly divided into the fission product (FP) decay heat accompanying the $\beta$-decay of the FP produced by nuclear fission and the actinide decay heat accompanying the $\alpha$-decay or $\beta$-decay of the actinide species, which are heavier than the uranium used as a nuclear fuel. There are several highly reliable methods that can be used to calculate the decay heat. Careful examination of the calculation method is necessary while performing the calculation for reasonably reproducing the situation of the nuclear reactor after its shut down.

\section{Introduction}

The most significant difference between nuclear fuel and fossil fuel lies in the fact that nuclear fuel continues to generate heat even after burning, i.e., even after the operation of the nuclear reactor has been terminated. Breakage or melting of the reactor core occurs if the nuclear fuel is not continually cooled. The heat remaining after reactor shutdown, which is caused by the delayed decay ( $\alpha$-and/or $\beta$-decay) of unstable nuclides produced during the operation of the reactor, is referred to as the decay heat. The time after reactor shutdown or the termination of burning can be referred to as the cooling time, which is the most important parameter related to decay heat. Decay heat is broadly divided into the fission product (FP) decay heat that accompanies the $\beta$-decay of the FP produced by nuclear fission and the actinide decay heat that accompanies the $\alpha$-decay or $\beta$-decay of the actinide nuclei, which are heavier than uranium, including plutonium, which is also used as fuel material.

\section{Fission Product (FP) Decay Heat}

\section{Origin and Characteristics of the FP Decay Heat}

A nucleus comprises both protons and neutrons. When the number of protons is expressed

DOI : 10.15669/fukushimainsights.Vol.1.88

(C) 2021 Atomic Energy Society of Japan. All rights reserved.

Originally published in Journal of the Atomic Energy Society of Japan (ISSN 1882-2606), Vol. 53, No. 8, p. 555-558 (2011)

in Japanese. (Japanese version accepted: May 30, 2011) 


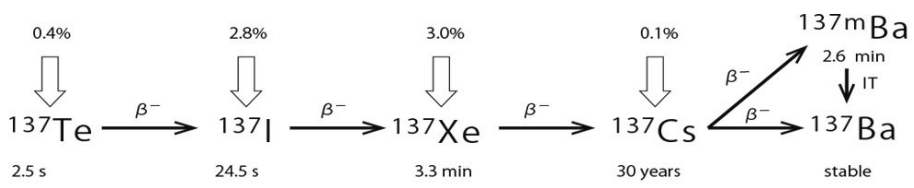

Figure 1 Example of $\beta$-decay chain (Mass number: 137)

as $\mathrm{Z}$ and the number of neutrons is expressed as $\mathrm{N}$, light nuclei often become stable at $\mathrm{N} / \mathrm{Z}=1.0$ (e.g., ${ }^{4} \mathrm{He},{ }^{10} \mathrm{~B}$, and ${ }^{12} \mathrm{C}$ ). As the atomic number $\mathrm{Z}$ of the nucleus increases, this $\mathrm{N} /$ $\mathrm{Z}$ ratio increases $\left(1.3\right.$ with ${ }^{93} \mathrm{Nb}, 1.4$ with ${ }^{133} \mathrm{Cs}$ ) and becomes $\mathrm{N} / \mathrm{Z}=1.6$ in case of uranium. Thus, heavy nuclei are observed to exhibit high ratio of neutrons. Therefore, when heavy ${ }^{235} \mathrm{U}$ undergoes nuclear fission and breaks up into two FPs having medium weight, both FPs are observed to contain excess neutrons. The FP that is unstable because of excess neutrons will repeat $\beta$-decay with time delay and move toward a stable nucleus while transforming neutrons into protons. Figure 1 depicts an example of this process. Because this process only transforms excess neutrons into protons, the mass number, $\mathrm{A}=\mathrm{N}+\mathrm{Z}$, does not vary. Further, such a $\beta$-decay chain can also be referred to as a mass chain.

This example depicts the situation in which the mass number is 137 . The white arrows in Figure 1 exhibit the generation of each nuclide due to nuclear fission; further, each number above them shows the generation rate of each nuclide (the numbers are relative to the ${ }^{235} \mathrm{U}$ thermal neutron fission). This rate is normalized at $200 \%$ and can be referred to as the independent yield. Each generated nuclide maintains its mass number, A, and continues its $\beta$-decay with the half-life that is written under the name of the nuclide until it reaches stable ${ }^{137} \mathrm{Ba}(\mathrm{N} / \mathrm{Z}=1.45)$. The total energies of the released $\beta$ - and $\gamma$-rays are considered to be the values of the decay heat. In case of FP decay heat, considering approximately 100 mass chains beginning from approximately $\mathrm{A}=75$ is necessary. The cumulative yield of ${ }^{137} \mathrm{Cs}$ that is frequently discussed is $6.3 \%$, which is the sum of the yields of the nuclides in the upstream (to the left of ${ }^{137} \mathrm{Cs}$ in the figure) and its own independent yield. This accumulated yield belongs to the largest category among all FPs. Though the energy of neutrinos, which inevitably accompanies the $\beta$-decay, is almost equivalent to those of the $\beta$ - or $\gamma$-rays, the neutrinos will still fly away to the outside of the universe without interacting with the substances around them. There are approximately 800 FP nuclides related to the FP decay heat. There are many FP nuclides having short lives that immediately contribute to the decay heat after shutdown; the number of FP nuclides contributing to the decay heat decreases with an increase in the cooling time.

\section{Summation of the FP Decay Heat}

The summation method is nearly an exclusive calculation method for calculating FP decay heat. The decay heat, $f(t)$, at cooling time, $t$, is obtained from

$$
f(t)=\sum_{i} \lambda_{i} \cdot\left(E_{\beta}{ }^{i}+E_{\gamma}{ }^{i}\right) \cdot N_{i}(t)
$$

This procedure is referred to as summation calculation because it utilizes the sum of all the FP nuclides. Here, $\lambda_{i}$ is the decay constant of nuclide $i$ and $N_{i}(t)$ is the abundance of nuclide $i$ at time $t . E_{\beta}{ }^{i}$ and $E_{\gamma}{ }^{i}$ denote the average energies of the $\beta$ - and $\gamma$-rays released when nuclide $i$ undergoes one $\beta$-decay. The FP decay data libraries, including the JNDC FP decay library ${ }^{1)}$, comprehensively include $\lambda_{i}, E_{\beta}{ }^{i}$, and $E_{\gamma}{ }^{i}$ together with detailed descriptions of their decay 
chains.

\section{The FP Decay Heat Time Behavior}

Figure 2 exhibits the FP decay heat of ${ }^{235} \mathrm{U}$. To simplify the explanation, we did not consider the effects of plutonium generation and combustion, which will be described later, and the minor actinide (MA) decay heat. The ratio of FP decay heat of ${ }^{235} \mathrm{U}$ after continuous burning for 2 years at constant power to the reactor operation power is shown. The recommendations provided by Japanese and USA institutions are observed to agree well each other. The FP decay heat observed immediately after shutdown is approximately $6.5 \%$ of the power while the reactor is being operated. Initially, the FP decay heat decreases rapidly and further continues to slowly decrease (note that it is a logarithmic axis.). It requires $8 \mathrm{~h}$ for the decay heat to become $1 / 10,4$ months to become $1 / 100$, and 3 years to become $1 / 1,000,000$ of the heat observed immediately after the shutdown. Furthermore, even after a decade of initiating burning shutdown, the level of FP decay heat is only 1/2,000 of the heat immediately after the shutdown. After a decade, even in case of uranium fuel, the heat emission from ${ }^{244} \mathrm{Cm}$ and ${ }^{238} \mathrm{Pu}$ in case of MA can no longer be ignored. This will be discussed later.

Finally, the influence of the difference in burning periods on the FP decay heat should be discussed. We observe that there are barely any differences between the decay-heat levels after a year of burning and after five years of burning; this can be observed immediately after the burning shutdown. However, their difference is observed to gradually increase by approximately two times after 120 days, approximately three times after a year, and more than four times after three years. Regardless, the decay heat level decreases along with an increase in the cooling time.

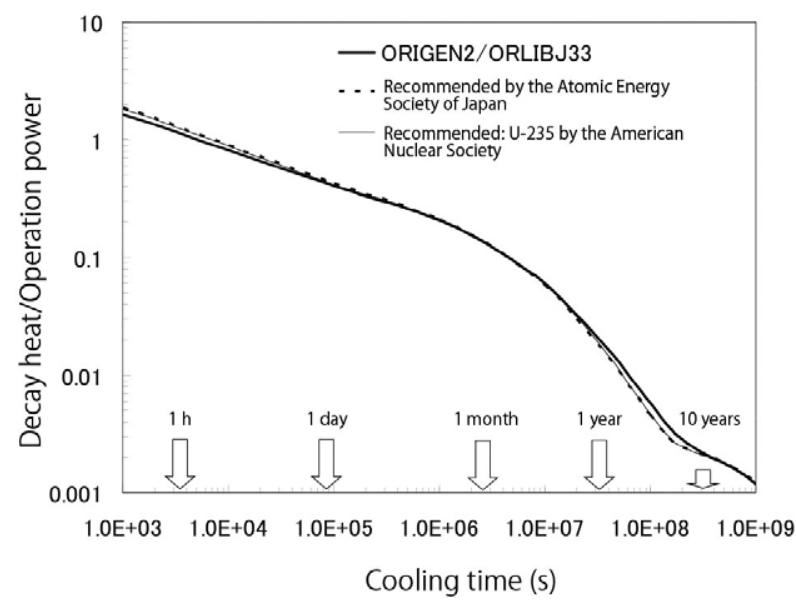

Figure 2 The FP decay heat of ${ }^{235} \mathrm{U}$ (after 2 year of operation)

\section{Behavior of the FP Nuclides and Decay Heat}

The number of the $i$-th nuclide $N i(t)$ that appears in equation (1) as a byproduct of the FP decay heat summation discussed in Section II-2 contains important information. Here, we present an example for this case in Figure 3, which depicts a comparison between the isotope 
number density ratio calculated using the cesium isotopic fractions in stagnated water from the Unit 2 turbine building of the Fukushima Daiichi Nuclear Power Plant released by Tokyo Electric Power Co., Inc. (TEPCO) in March, 2011 and the isotope ratio Ni ( $t$ ) calculated using ORIGEN2 code, which will be discussed later. The calculated and measured values are observed to agree well. Among these, ${ }^{137} \mathrm{Cs}$ is a typical FP nuclide that also appeared in Figure 1 ; however, ${ }^{134} \mathrm{Cs}$ exhibits completely different characteristics. The cumulative yield of ${ }^{134} \mathrm{Cs}$ from the ${ }^{235} \mathrm{U}$ thermal neutron fission is $7 \times 10^{-6} \%$, which is substantially equal to none. In truth, this isotope was generated by ${ }^{133} \mathrm{Cs}$ (cumulative yield 6.7\%), which was accumulated as the terminal stable isotope of the $\beta$-decay chain in the mass chain of $\mathrm{A}=133$ while the nuclear reactor was in operation, capturing the neutrons in the reactor. ${ }^{134} \mathrm{Cs}$ is generated as though "waking a sleeping dog," and it increases the FP decay heat by several dozens in percentage with 1-4 years of cooling time. There are several other nuclides that behave in a similar

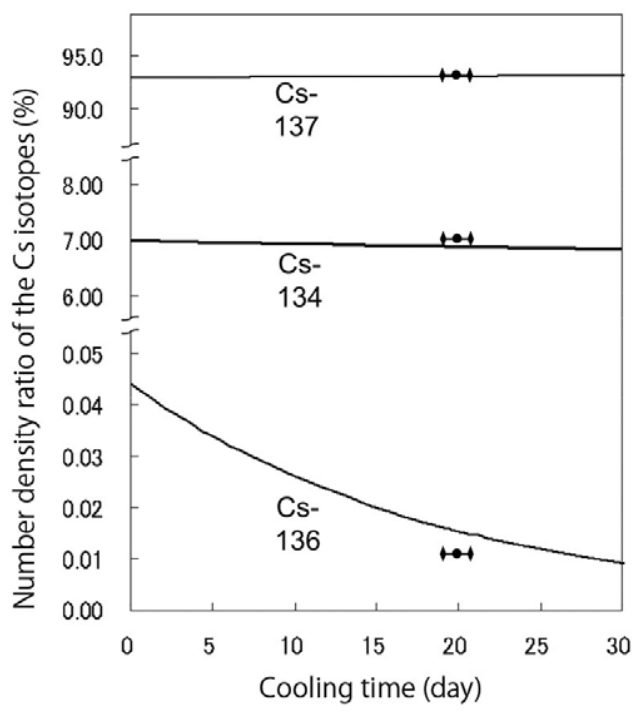

Figure 3 The Cs isotope number density ratio of the stagnated water in unit 2 turbine building (calculated by ORIGEN2/ORLIBJ 33)

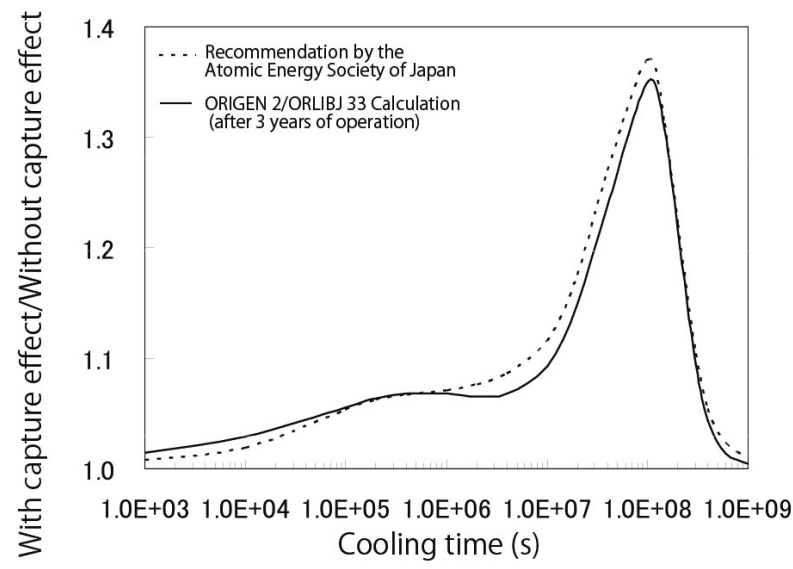

Figure 4 Neutron capture effect in the FP decay heat of ${ }^{235} \mathrm{U}$ 
manner. This effect can be referred to as the neutron capture effect. The large peak that appears at an approximate cooling time of $10^{8}$ s (approximately 3 years), depicted in Figure 4, can be attributed to ${ }^{134} \mathrm{Cs}$. This is observed to increase the decay heat by more than $30 \%$.

Table 1 presents the top ten nuclides that contribute to the FP decay heat of ${ }^{235} \mathrm{U}$ at Units 1 to 3 of the Fukushima Daiichi Nuclear Power Plant at the point of publication of this study (scheduled for August 1), which is approximately 140 days since the reactor shutdown (March 11, 2011). These nuclides correspond to the top ten nuclides of the sum of equation (1). Among them, only four types of nuclides, namely ${ }^{144} \mathrm{Pr},{ }^{95} \mathrm{Nb},{ }^{106} \mathrm{Rh}$, and ${ }^{95} \mathrm{Zr}$, produce $76 \%$ of the ${ }^{235} \mathrm{U}$ FP decay heat; further, the sum of the heats of all ten nuclides contribute to $96 \%$ of the heat. Note that the half-life of ${ }^{144} \mathrm{Pr}$, which is on the top of the list, is only $17.3 \mathrm{~min}$. A nuclide with such a short half-life continues to exhibit an effect even 5 months after the reactor shutdown because ${ }^{144} \mathrm{Ce}$, which has a long half-life of 285 days, is located immediately before ${ }^{144} \mathrm{Pr}$ in the mass chain having a mass number of 144 , which delays the generation of ${ }^{144} \mathrm{Pr}$.

Table 1 FP nuclides with largest contribution to the decay heat of ${ }^{235} \mathrm{U}$ (after 140 days of cooling)

\begin{tabular}{ccc}
\hline Ranking & Nuclide & Contribution $(\%)$ \\
\hline 1 & ${ }^{144} \mathrm{Pr}$ & 34.0 \\
2 & ${ }^{95} \mathrm{Nb}$ & 17.8 \\
3 & ${ }^{106} \mathrm{Rh}$ & 13.7 \\
4 & ${ }^{95} \mathrm{Zr}$ & 10.1 \\
5 & ${ }^{91} \mathrm{Y}$ & 4.9 \\
6 & ${ }^{134} \mathrm{Cs}$ & 3.7 \\
7 & ${ }^{144} \mathrm{Ce}$ & 3.0 \\
8 & ${ }^{89} \mathrm{Sr}$ & 2.8 \\
9 & ${ }^{103} \mathrm{Ru}$ & 2.0 \\
10 & ${ }^{90} \mathrm{Y}$ & 2.0 \\
\hline
\end{tabular}

\section{Actinide Decay Heat}

\section{Generation of Plutonium}

Even in case of a light-water reactor, the neutron capture reaction of ${ }^{238} \mathrm{U}$ continues while the uranium fuel is burning. The generated ${ }^{239} \mathrm{U}$ becomes ${ }^{239} \mathrm{Pu}$ after two steps of $\beta$-decay are completed in the following manner.

$$
{ }^{238} \mathrm{U}(n, \gamma){ }^{239} \mathrm{U}(23.5 \mathrm{~min}) \rightarrow{ }^{239} \mathrm{~Np}(2.36 \text { day }) \rightarrow{ }^{239} \mathrm{Pu}
$$

The times attached to both the nuclides denote their half-lives. Because these two $\beta$-decays are accompanied by a time delay, it is considered to be a part of the decay heat. The decay heat generated by this process can be referred to as U-Np decay heat to differentiate it from the MA decay heat that will be discussed in the subsequent section.

\section{Minor Actinide Decay Heat}

Even with a light-water reactor, MAs, including ${ }^{238} \mathrm{Pu}$, neptunium, americium, and curium, 


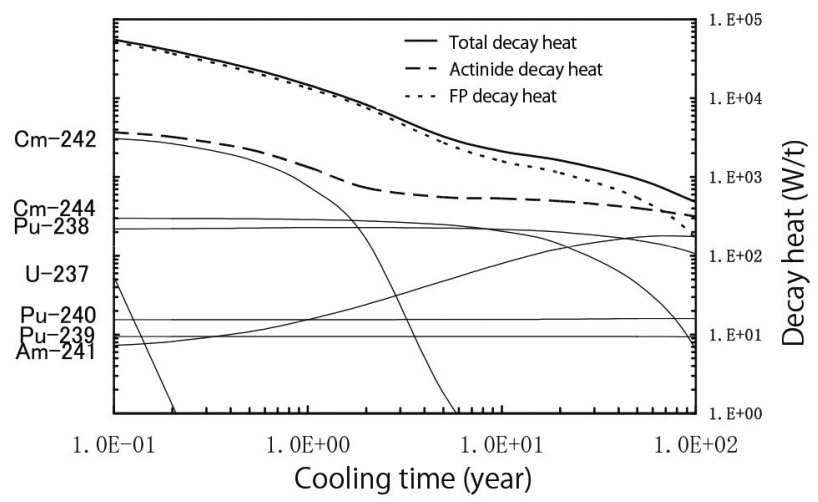

Figure 5 FP and actinide decay heats of uranium fuel after the shutdown of the operation

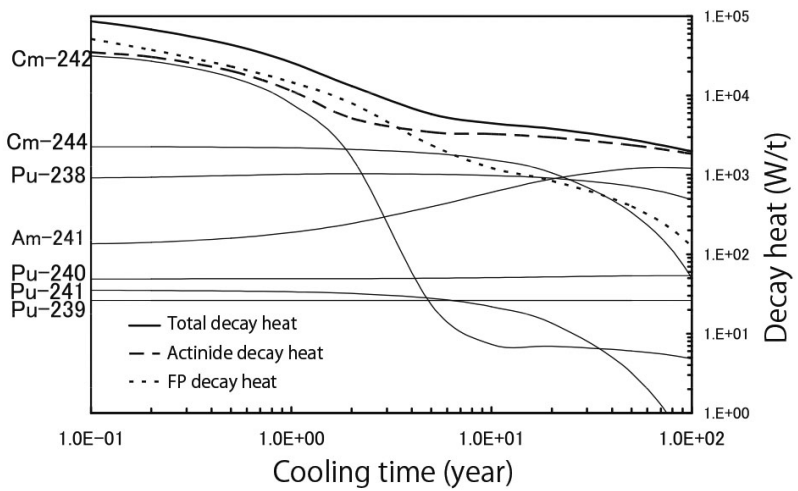

Figure 6 FP and actinide decay heats of MOX fuel after the shutdown of the operation

are accumulated after a long period of operation, and the MA decay heat that accompanies either the $\alpha$ - or $\beta$-decay of these nuclides can no longer be ignored. Figures 5 and $\mathbf{6}$ depict the contents of the FP and MA decay heats of uranium fuel decay heat and MOX fuel decay heat, respectively. As mentioned in the previous section, the U-Np decay heat is not included in these figures.

In case of the spent uranium fuel, the FP decay heat is observed to be dominant until a few years of cooling time, and the actinide decay heat is observed to decrease by approximately one decade. However, their positions in terms of size are reversed after a little less than a century of cooling time. In case of light-water reactor MOX fuel, their difference is observed to be small from the beginning; further, they become approximately identical after a few months of cooling time. The actinide decay heat is observed to become dominant after a few years.

\section{Calculation/Evaluation of the Decay Heat}

This chapter discusses the standard methods used to calculate/evaluate the decay heat. 


\section{Recommendations by Public Organizations}

(a) Recommendation by the Atomic Energy Society of Japan (AESJ) ${ }^{2)}$ : This is the recommendation by the "Nuclear reactor decay heat standard" special research committee of AESJ. The FP decay heat after instantaneous irradiation is expressed as an exponential polynomial (33 terms) with respect to the cooling time, $t$. The $66(33 \times 2)$ constant values that appear in the equation are assigned to each fuel nuclide and provided in reference 3 ). The decay heat after a given time of the operation with constant power can be obtained by integrating the decay heat of instantaneous irradiation with respect to time $t$; however, the result of this integration also becomes a simple exponential polynomial. Thus, it can be easily calculated, for example by using EXCEL. This is one of the advantages of the use of an exponential polynomial. For the neutron capture effect discussed in Section II-4, a correction factor is given, whereas an evaluation equation is given for the U-Np decay heat discussed in III-1. Furthermore, the MA decay heat must be separately calculated.

(b) Recommendation by the American Nuclear Society (ANS) ${ }^{4}$ : This is the result of consideration and evaluation of the summation result and the nuclear-fuel-sample irradiation experiment result by the ANS 5.1 working group of the ANS Standard Committee. The FP decay heat curve after instantaneous irradiation is expressed through exponential polynomials having 23 terms. This recommendation is reviewed and revised every few years and is well maintained. The treatment of the neutron capture effect, the U-Np decay heat, and the MA decay heat is the same as in the case (a) above.

\section{Appropriate Usage of the Summation Code and Attached Library}

(a) Usage of the OREGEN2 Code ${ }^{5}$ : The OREGEN2 code is extensively used worldwide because it is easy to use and highly useful owing to its clear input and comprehensive output. However, the calculation result may be altered because of the nuclear data library. The recommended usage of ORLJBJ-33 ${ }^{6}$ ) is based on the JENDL 3.3 nuclear data library, which has already been evaluated in Japan, and the JNDC FP decay data library ${ }^{1}$. Regarding the FP decay heat, the recommendation by AESJ discussed in IV-1 can be recreated by strictly matching the calculation condition using ORLJBJ-33. The MA decay heat, which cannot be easily evaluated using IV-1(a) and (b) methods, naturally enters the calculation. Additionally, during the late phase of burning the uranium fuel, the fission heat of plutonium becomes $30 \%-40 \%$ of the entire nuclear heat. Therefore, the situation for FP decay heat is observed to be different from the time when only ${ }^{235} \mathrm{U}$ contributed to the nuclear fission. OREGEN2 is also able to easily reflect this effect in the calculation.

\section{Measurement of the Decay Heat}

There are many data sets of directly measured decay heat. Typical examples include the measurements by Akiyama et al. of Tokyo University and by Dickens et al. of Oak Ridge National Laboratory in the USA. Both groups irradiated pure samples of ${ }^{235} \mathrm{U},{ }^{239} \mathrm{Pu}$, and so on, using neutrons in the experimental reactor core and removed; further, they measured the $\beta$-ray components using a plastic scintillator and the $\gamma$-ray components using a NaI (TI) scintillator and integrated them with energy to obtain the $\beta$ - and $\gamma$-ray components of the FP decay heat. These two sets of measured results, converted to those for time $t$ after a fission burst, were substantially referred to and reflected while preparing the recommendations by public 
organizations discussed in IV-1. Note that the Japanese and American recommendations are very similar, as depicted in Figure 2. These two recommendations were both independently evaluated. The fact that both were similar enhances the reliability of the recommendations. However, it must be remembered that both the recommendations are based on the same measurements, two of which were described above as examples. Therefore there exists a possibility that both may suffer from the same systematic error that originated from the measurements as part of their basis. Unfortunately, such sample-irradiation experimental data only exist up to $6 \mathrm{~h}$ of cooling time (measurement conducted by the Tokyo University). One of the few measurements required to ensure long-term cooling is the measurement at the fast experimental reactor, "Joyo," conducted by Aoyama et al. They sealed the reactor fuel assembly in a specially constructed calorimeter and directly measured the decay heat during 24-258 days after completing the burning. However, despite the detailed analytical calculation based on the JENDL 3.2 and JNDC FP decay data library, the measurement and calculation were not observed to satisfactorily agree. It is reported that the calculation exhibits an underestimation by approximately $10 \%^{7)}$. Regarding the calculated value of the decay heat, it should be understood that, after long-term cooling, a prediction error of approximately $10 \%$ should be expected for a while. However, the problem that the decay heat estimation of long-term cooling is excessively dependent on summation remains owing to the lack of actual measurement data.

\section{Conclusions}

Calculating the decay heat of a nuclear reactor is relatively easy. Additionally, the reliability of this calculation is guaranteed to a certain extent, and the error evaluation results are published. While evaluating or calculating the decay heat, it is necessary to carefully examine the calculation methods and verify whether they achieve the required accuracy. Are MA components included? How is the neutron capture effect of FP treated? In some cases, noble gas components, such as $\mathrm{Kr}$ or $\mathrm{Xe}$, or components that easily dissolve in water, such as I or Cs, may have already escaped from the fuel. Hence, ascertaining the condition of the nuclear reactor after shutdown is necessary while performing the evaluation.

Finally, I am deeply grateful to Mr. Hiroto Matsumoto, a 2nd year student of the master's course of the Tokyo City University who dedicated his time and cooperated in preparing this article.

\section{References}

1) Tasaka K. et al., JNDC Nuclear Data Library of Fission Products, JAERI 1287, 1983, Japan Atomic Energy Agency.

2) Special research committee of AESJ - Nuclear Reactor Decay Heat Standard, Nuclear Reactor Decay Heat and Recommendation for It, 1989, Atomic Energy Society of Japan. [in Japanese]

3) Special research committee of AESJ - Nuclear Reactor Decay Heat Standard, Decay Heat Recommendation and How to Use It, 1990, Atomic Energy Society of Japan. [in Japanese]; English translation available: Tasaka K. et al, JAERI-M 91-034, 1991, Japan Atomic Energy Agency.

4) American National Standard. Decay Heat Power in Light Water Reactors, 2005, ANSI/ANS-5.1-2005, American Nuclear Society.

5) Croff A.G., Nucl. Sci. Eng., 62, 1980, pp.335-250.

6) Katakura J. et al., JAERI-Data/Code 2002-21, 2002, Japan Atomic Energy Agency. [in Japanese]

7) Aoyama T. et al. Nihon-Genshiryoku-Gakkai Shi (J. At. Energy Soc. Jpn), 41, 1999, pp. 946-953. [in Japanese] 